\title{
Electroluminescence from GaN Nanostructures
}

\author{
Alemayehu Keno ${ }^{1}$, Getnet Melese ${ }^{2, *}$, L.V Choudary ${ }^{2}$ \\ ${ }^{1}$ Nekemte College of Teachers' Education, Ethiopia \\ ${ }^{2}$ Department of Physics, Jimma University, Ethiopia
}

Copyright (C) 2015 Horizon Research Publishing All rights reserved.

\begin{abstract}
Electroluminescence (EL) intensity from GaN nanostructures is reported as a function of different parameters, such excitation wavelength, number of the nanostructures, applied voltage, temperature and time. Quantum confinement model (QCM) is used to develop the model equation that describes the EL intensity as a function of size of the nanostructures. It is shown that as the number of nanostructures decreases EL intensity increases. The highly efficient EL intensity is obtained at low operating voltage $(6 \mathrm{~V})$. It is observed that EL intensity decreases as the temperature increases and degrades with time.
\end{abstract}

Keywords Gallium-nitride, Nanostructures, Quantum Confinement, Recombination, Electroluminescence

\section{Introduction}

In studying properties of a semiconductor quantum confinement (QC) is important. It is the electrons confinement in semiconductors along the spatial coordinates $[1,2]$. Quantum dots (QDs) are semiconducting nanoparticles that are able to trap electrons in small spaces. QDs confine electrons, holes, or electron-hole pairs to zero dimensions of the order of electrons de Broglie wavelength. They contain a single unit of charge and give off different colors of light depending on size and specific energy levels [3].

Semiconductor QDs have rich optical properties that strongly depend on size, especially when the particle size is less than the exciton Bohr radius of the material $[4,5]$. The electrical and optical properties of group III nitride materials are of great interest for light-emitting diodes (LEDs), lasers and other optoelectronic devices because of their band structures and the large range of band gap (0.7-6.2 eV). GaN with a band gap of $3.4 \mathrm{eV}$ has received a great deal of attention for its blue and ultraviolet light emission and because of its application in high-temperature, high-power devices [6].

EL has been reported for porous silicon during anodic oxidation, and also for porous silicon devices employing thin gold, indium tin oxide, silicon carbide, and polymer contacts.
It is a light emission phenomenon caused by the electric current or strong electric field passing through a material. When electrons and holes recombine in the material, the energy of the excited electrons will be released in the form of photons $[7,8]$. The main objective of this work is to study the EL intensity of GaN nanostructures as a function of excitation wavelength, applied voltage, temperature and time.

\section{Dependence of EL Intensity on Different Parameters}

Semiconductors are transparent to photons whose energies lie below their band gap and strongly absorb photons whose energies exceed the band gap energy. When light passes through a material, the light is partially scattered, absorbed, or transmitted. The transmitted beam intensity decreases exponentially with the specimen thickness and absorption coefficient. The absorption coefficient $\alpha$ is a property of material which defines the amount of light absorbed by it. The transmitted light intensity as a function of the effective thickness of the sample $z \quad[9,10]$ :

$$
I(z)=I_{o} e^{-\alpha . Z}
$$

Where I is the intensity of incident light.

EL intensity as a function of temperature with intensity at absolute zero temperature $I_{0}$ can be fitted by a standard equation [11]

$$
I_{E L}(T)=\frac{I_{o}}{1+C_{1} \exp \left(\frac{-E_{1}}{K_{B} T}\right)+C_{2} \exp \left(\frac{-E_{2}}{K_{B} T}\right)}
$$

Where $E_{1}$ and $E_{2}$ are the two thermal quenching activation energies, $T$ is the temperature, $K_{B}$ is the Boltzmann constant $C_{1}$ and $C_{2}$ are coupling coefficients. The EL thermal quenching activation energy of GaN is $190 \mathrm{meV}$ [12].

The radiative and non-radiative processes occurring in the active layer containing $\mathrm{GaN}$ nanoclusters have been studied by means of time-resolved luminescence measurements. Time resolved measurements of the EL signals have been 
extracted by fitting the experimental curves with the equation given by $[13,14]$ :

$$
I(t)=I_{o} \exp \left[-(t / \tau)^{\beta}\right]
$$

Where $I(t)$ and $I_{0}$ are the EL intensity as a function of time $t$ and at time $t=0 \mathrm{sec}$, respectively, $\tau$ is the EL decay time constant and $\beta$ is a dispersion factor [15].

\section{Methods and Formulation of Models for $E L$}

\subsection{Methods}

We designed computational method which is based on theoretical study and numerical analysis of EL intensity from $\mathrm{GaN}$ nanostructures as a function of different parameters. We developed model equation that describes the EL intensity as a function of size of the nanoclusters using quantum confinement model and used Fourier transform to transform the size dependent intensity into energy dependent intensity. After we have derived the model equation as a function of energy, we developed suitable MATLAB code for our master equation to generate data and plot the graphs in different legends.

\subsection{The Quantum Confinement Model (QCM)}

Quantum confinement is a very successful model for understanding the electronic structure of nano-size semiconductor structures. It known that with decreasing particle sizes the band gap increases due to shifting of the band edges [16].

Assuming a Gaussian model of size distribution about the mean diameter $\mathrm{L}_{0}$ for the nanocrystallites, the size dependent intensity is given by [17];

$$
I(L)=\frac{1}{\sqrt{2 \pi} \sigma} \exp \left(-\frac{\left(L-L_{o}\right)^{2}}{2 \sigma^{2}}\right)
$$

Where $L$ is the cluster diameter and $\sigma$ is the standard deviation of the diameter.

The number of carriers $\left(\mathrm{N}_{\mathrm{c}}\right)$ in a column of diameter' $L$ 'participating in the EL process is proportional to $\mathrm{L}^{2}$ and hence it is given by:

$$
N_{c} \approx L^{2} \Rightarrow N_{c}=a L^{2}
$$

Where, " $a "$ is suitable normalization constant taken as 1 in our simulation.

For a GaN sample consisting of varying column diameters the probability distribution of electrons participating in the EL process is:

$$
I(L)=\frac{1}{\sqrt{2 \pi} \sigma} a L^{2} \exp \left(-\frac{\left(L-L_{o}\right)^{2}}{2 \sigma^{2}}\right)
$$

The luminescence intensity can be determined by the Fourier transform of equn.(6) to the energy axis as:

$$
I(\Delta E)=\int_{0}^{\infty} \frac{1}{\sqrt{2 \pi} \sigma} a L^{2} \delta\left(\Delta E-\frac{C_{1}}{L^{2}}\right) \exp \left(-\frac{\left(L-L_{o}\right)^{2}}{2 \sigma^{2}}\right) d L
$$

Where $C_{1}$ is constant taken as $0.00485816 \mathrm{Jm}^{2}$ in our simulation.

The Dirac delta function facilitates a straight forward integration. Simplifying and applying the properties of Dirac delta function, the above integral gives the intensity as a function of $\Delta \mathrm{E}$.

$$
I(\Delta E)=\frac{a C^{\frac{3}{2}}}{\sqrt{8 \pi} \sigma} \Delta E^{\frac{-5}{2}} \exp \left(-\frac{L_{o}{ }^{2}}{2 \sigma^{2}}\left[\left(\frac{\Delta E_{o}}{\Delta E}\right)^{\frac{1}{2}}-1\right]^{2}\right.
$$

Where $\Delta E_{0}=\frac{C_{1}}{L_{0}^{2}}$ and $\Delta E$ the energy shift due to the confinement.

Thus, eqn(8) which is transformed from eqn (6) is the main focus of this work and is our model equation.

The energy shift due to confinement is given by:

$$
\Delta E=h v-\left(E_{g}-E_{b}\right)
$$

Where $\mathrm{h}$ is Planck's constant, $v$ is the excitation frequency of light, $E_{g}$ is the bulk band gap of GaN and $E_{b}$ is the exciton binding energy. Depending on temperature the values of $E_{g}$ ranges from $3.39 \mathrm{eV}$ to $3.52 \mathrm{eV}$; however, we took $3.4 \mathrm{eV}$ which is actually reported in most standard experiments at room temperature, while the value of $E_{b}$ varies with nanocrystal radius of the sample. For GaNnanostructures it is reported in the range of $20.4 \mathrm{meV}$ to $28 \mathrm{meV}$ and $25.6 \mathrm{meV}$ is used for our simulation which is the experimental value as reported in literature $[11,18,19]$.

The EL spectrum is caused by injection of strong electric field $\mathbf{E}$ or current, the energy associated to this field is:

$$
\varepsilon=N Z e E L=N Z V e
$$

where $\mathrm{N}$ is the number of nanocrystallites of $\mathrm{GaN}, Z$ is the number of electrons in each crystal, $e$ is the charge of electron and $E$ is the magnitude of external applied electric field which is responsible for EL and in the direction of diameter, ' $L$ ' is the diameter of the single nanocrystal and $V$ is the voltage applied to the nanocrystal sample. Since EL and PL occurs at the same energy, using the analogy of the PL spectrum model we can write the energy shift due to electrical excitation as:

$$
\Delta E=N Z V e-\left(E_{g}-E_{b}\right)
$$

According to the QC model, the emission wavelength and intensity depends on nanocrystal diameter, size distribution and concentration [20,21].

\section{Results and Discussion}




\subsection{Intensity Versus Wavelength}

Fig (1) shows the EL intensity as a function of excitation wavelength for $\mathrm{GaN}$ nanostructures. Our computational result is in agreement with the experimental result of M. G. Cheonga et al [22].

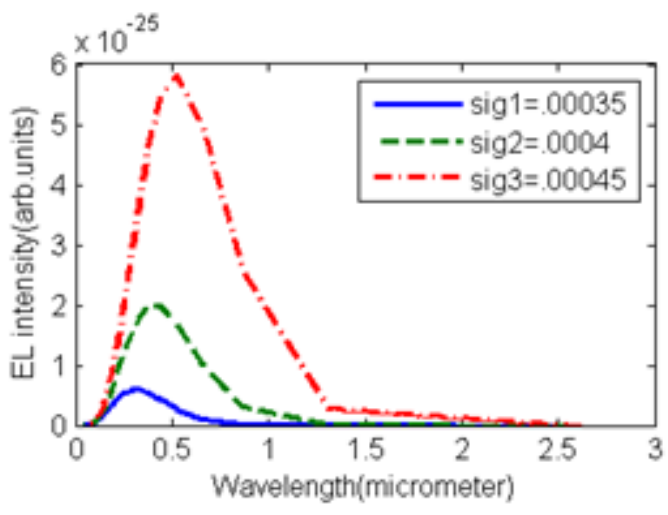

Figure 1. EL intensity versus excitation wavelength for different size of $\mathrm{GaN}$ nanostructures

In both cases the EL intensity has sharp peak near the center of the Gaussian curve. The figure shows that as wavelength increases the intensity maxima shifts to lower energy (red-shifted) and as we go further maximum intensity becomes out of the visible range. The higher value of standard deviations suppresses the nature of EL intensity that would have been observed for the smallest standard deviations.

\subsection{Variation of Intensity with Number of GaN Nanostructures and Applied Voltage}

Fig (2a) shows EL intensity as a function of number of $\mathrm{GaN}$ nanostructures for different fixed values of applied voltage. Decreasing the number of nanostructures in the sample decreases the diameter of the nanocluster. This increases the luminescence intensity. When the size of nanocluster decreases, the surface-to-volume ratio increases. This causes quantum confinement effect. The band gap energy increases due to quantum confinement, which enhances radiative recombination rate of excitons for emission of light.

Higher EL intensity is obtained for small number of nanostructures as depicted in figure (2a). It is shown that for too large or too small number of nanostructures for a fixed voltage, the EL spectrum will lie in the ultraviolet or infrared region respectively.

Figure (2b) shows EL intensity as a function of voltage for different fixed number of GaN nanostructures. EL intensity increases as the applied voltage increases; however, to get spectrum in the visible region and for better quantum efficiency low voltage $(<6 \mathrm{~V})$ is needed. We get maximum average intensity when the applied voltage is about $6 \mathrm{~V}$. After a certain maximum peak the EL intensity decreases infinitely with increasing value of the applied voltage.

The causes for this effect may be carrier linkage at high forward currents, Auger recombination, or junction heating carrier delocalization.

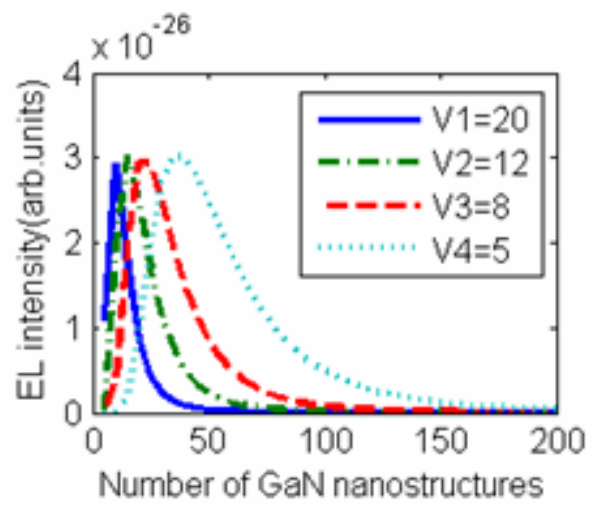

\section{a) EL intensity Vs Number of GaN structures}

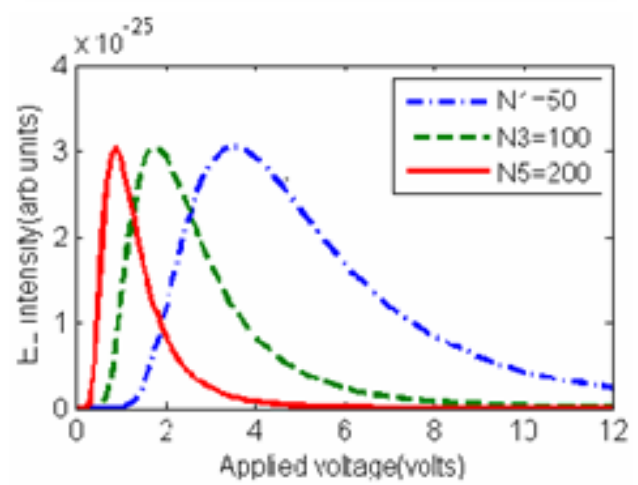

b) EL intensity Vs Applied voltage

Figure 2. EL intensity as a function of a) number of $\mathrm{GaN}$ nanostructures $b$ ) applied voltage

\subsection{EL intensity Versus Temperature}

Fig (3) depicts the simulated result of eqn (2) which shows the dependence of EL intensity on the temperature for $\mathrm{GaN}$ nanostructures. As temperature increases EL intensity decreases. This is due to high absorption and the shrinkage of band gap with increasing temperature. The EL quenching at increased temperature is a combination of a reduced confinement and thermally activated non-radiative recombination processes within "active" crystallites.

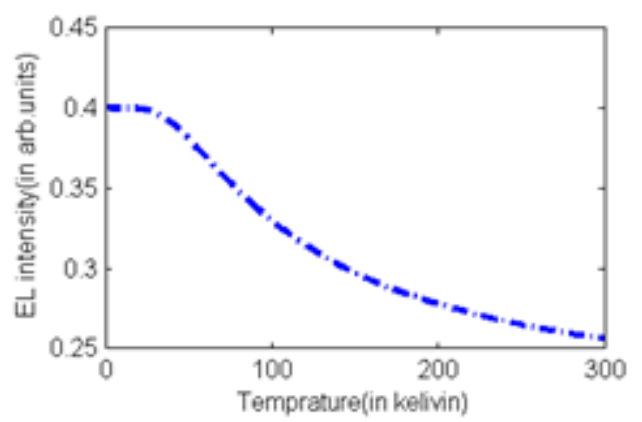

Figure 3. EL intensity as a function of temperature for $\mathrm{GaN}$ nanostructures 


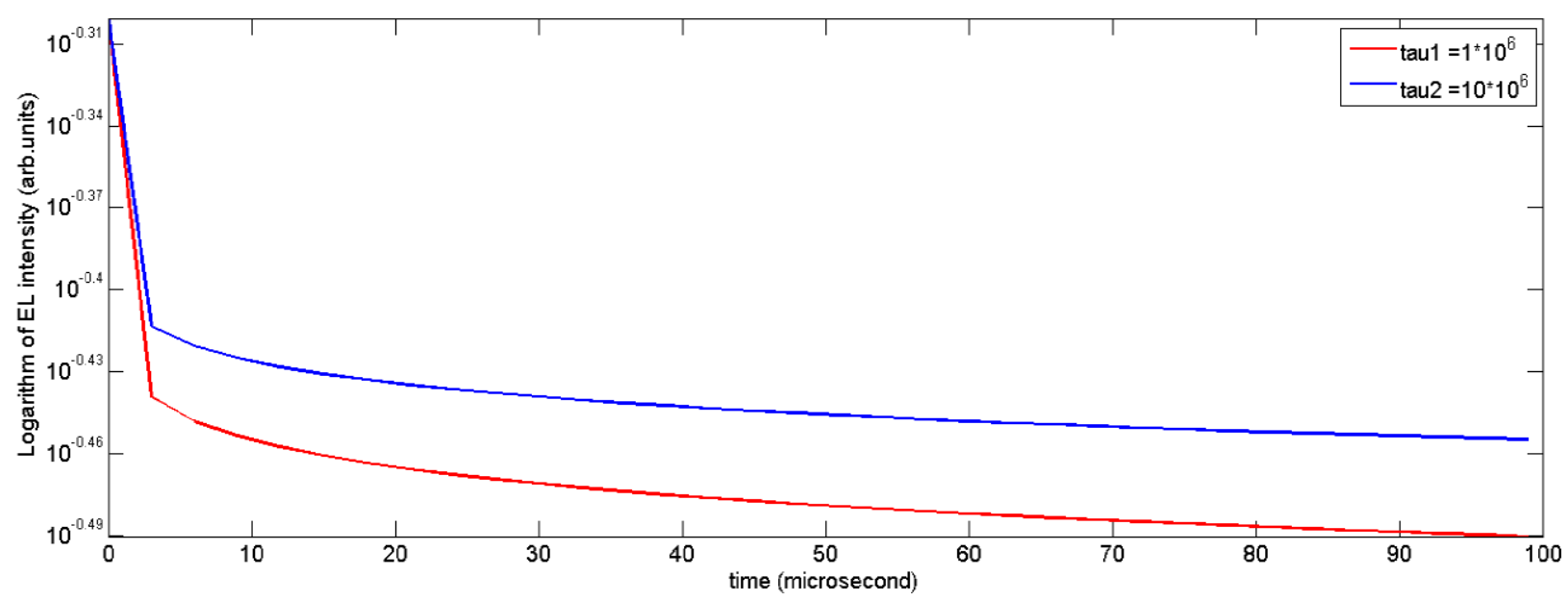

Figure 4. Logarithm of EL intensity as a function of time for GaN nanostructures

\subsection{EL Intensity Versus Time}

Fig (4) illustrates the simulated result of eqn (3) which shows the time dependence of EL intensity. Our result is much closer to experimental result of NenadLalic [14]. A shorter decay time constant allows a higher modulation frequency, but reduces the efficiency. This is due to the decrease in oscillation period and non-radiative recombination of the particles in the nanocrystal.

This transient property indicates that EL shows either reversible or permanent degradation with time.

\section{Conclusions}

EL spectrum has Gaussian sub peaks and red shifted with increasing wavelength, nanocrystals size and applied voltage. A blue shift of EL is observed with decreasing nanocluster size and applied voltage. High EL intensity is obtained for small number of nanostructures and light emission starts at low threshold voltage. EL shows either reversible or permanent degradation with time. It usually increases at lower temperature due to inhibition of electron-phonon interactions and thereby increases the excited electronic state lifetime.

\section{Acknowledgements}

We are grateful to Jimma University and Nekemte College of Teachers' Education for their financial support.

\section{REFERENCES}

[1] William E. Buhro and Vicki L. Colvin, Semiconductor nanocrystals. Nature PublishingGroup (2003).

[2] Harrison Paul, Quantum wells, wires and dots, 2nd edition, John Wiley and Sons Ltd.,2005.
[3] Charles Kittel, Introduction to solid state physics 8th edn., John Wiley and Sons, ,Inc. New York, (2005).

[4] Emile Knystautas Boca, Engineering thin films and nanostructures with ion beams. Taylor and Francis, New York, (2005).

[5] Jin Z. Zhang and Christian D. Grant, Optical and dynamic properties of undoped and doped semiconductor nanostructures, Annual Review of Nano Research, World Scientific Publishing Co. Pte.Ltd. (2007).

[6] Birgit Schwenzer et al, Gallium nitride powders from ammonolysis: Influence ofreaction parameters on structure and properties, Appl.phys.Lett. 16, 5088-5095, (2004).

[7] K. V. Shcheglov et al, Electroluminescence and photoluminescence of ge-implanted $\mathrm{Si} / \mathrm{SiO}_{2} / \mathrm{Si}$ structures. Appl. Phys. Lett. 66 (1995).

[8] Ben G. Streetman and Sanjay Kumar Banerjee, "excess carriers in semiconductors."'in solid state electronic devices, Pearson Prentice Hall, 6th edn, (2006)

[9] TaiCheng Tsai et al, Electroluminescence emission of crystalline silicon nanoclustersgrown at low temperature, Nanotechnology 18, 275707, (2007).

[10] E. Fred Schubert, Light-emitting diodes. Cambridge University Press, 2nd.edition, 2006.

[11] S. H. Na and K. Kyhm, Time-resolved free exciton trapping and thermal activationin bound-exciton in GaN., Journal of the Korean Physical Society 51, 539-544, (2007).

[12] II-Kyu Park et al, Ultraviolet light-emitting diodes with self-assembled ingan quantumdots.Appl. Phys. Lett.90, 111116), (2007).

[13] Alessia Irreral et al, Electroluminescence and transport properties in amorphous silcon nanostructures, Nanothechnology17, 1428-1436 (2006).

[14] NenadLalic, Light emitting devices based on silicon nanostructures, KTH, RoyalInstitute of Technology, Sweden (2000).

[15] Yoshihiko Kanemitsu, Luminescence properties os nanometer-sized si crystallites:Core and surface states, Physical Review B 49, 16845-16848 (1994). 
[16] T. M. Willey et al, Molecular Limits to the QuantumConfinement Model in Diamond Clusters, Physical Review Letters,95, (2006).

[17] P. F. Trwoga et al, Modeling the contribution of quantum confinement to luminescencefrom siliconnanoclusters, J. Appl. Phys. 83, 3789-3794, (1998).

[18] Youfan $\mathrm{Hu}$ et al, Piezo-phototronic effect on electroluminescence properties of p-type $\mathrm{GaN}$ thin films, Nano Letters (2012).

[19] Seoung-Hwan Park et al, Exciton binding energy in wurtziteInGaN / GaN quantumwells, Journal of the Korean
Physical Society, 45, 582-585, (2004).

[20] Tekchandaniet al, Preparation, characterization and electroluminescence studies of cadmium selenidenanocrystals, The Open Nanoscience Journal,5, 59-63, (2011).

[21] D. R Vij, Hand book of electroluminescent materials, Institute of physics, GreatBritian, 2004.

[22] M.G. Cheonga, et al. Properties of InGaN/GaNquantum wells andblue light emitting diodes. Journal of Luminescence, 99,265-272, (2002). 